\title{
A Few Shot Adaptation of Visual Navigation Skills to New Observations using Meta-Learning
}

\author{
Qian Luo ${ }^{1}$, Maks Sorokin ${ }^{1}$, Sehoon $\mathrm{Ha}^{12}$
}

\begin{abstract}
Target-driven visual navigation is a challenging problem that requires a robot to find the goal using only visual inputs. Many researchers have demonstrated promising results using deep reinforcement learning (deep $R L$ ) on various robotic platforms, but typical end-to-end learning is known for its poor extrapolation capability to new scenarios. Therefore, learning a navigation policy for a new robot with a new sensor configuration or a new target still remains a challenging problem. In this paper, we introduce a learning algorithm that enables rapid adaptation to new sensor configurations or target objects with a few shots. We design a policy architecture with latent features between perception and inference networks and quickly adapt the perception network via meta-learning while freezing the inference network. Our experiments show that our algorithm adapts the learned navigation policy with only three shots for unseen situations with different sensor configurations or different target colors. We also analyze the proposed algorithm by investigating various hyperparameters.
\end{abstract}

\section{INTRODUCTION}

Visual navigation is a fundamental skill for robotic creatures to conduct real-world autonomous missions, such as logistics, surveillance, or home-assistance. However, learning visual navigation policy is a challenging problem that involves multiple aspects of robotics research, from scene understanding to motion planning. Recent advances in deep learning have enabled robotic agents to learn effective navigation policies in various scenarios [1], [2], [3]. However, deep RL often requires a tremendous amount of computational costs. For instance, the work of Wijmans et al. [4] requires a large GPU cluster with 64 nodes to solve a point-to-point navigation problem, which corresponds to sixmonths of GPU time.

Learning visual navigation becomes even more challenging if we start to consider a lot of heterogeneous robots needing navigation skills for their missions, such as wheeled robots for warehouse logistics, legged robots for wilderness monitoring, or drones for last-mile delivery. A naive approach is to learn a navigation policy for each robot independently, but it will be computationally expensive. To make matters worse, there is no guarantee that the same scheme will lead to the same successful policy on all the robots. An alternative approach is to transfer a learned policy from one robot to another robot: however, heterogeneous robots have different sensing and actuation capabilities, making the transfer difficult. Figure 1 illustrates camera inputs of different robots(different sensor heights) that are sampled

\footnotetext{
${ }^{1}$ Georgia Institute of Technology, Atlanta, GA, 30308, USA

2 Robotics at Google, Mountain View, CA, 94043, USA

Emails: \{luogian, maks, sehoonha\}@gatech.edu
}

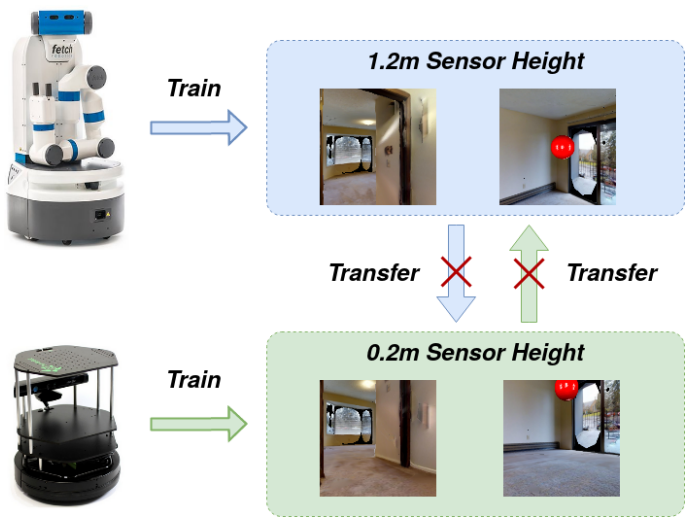

Fig. 1. The visual features of robots of different sensor heights are quite different, making it hard to directly transfer the learned policy to another robot. Visual navigation for heterogeneous robots must resolve perspective differences.

from the same location, which present noticeable different visual features.

In this work, we propose a novel algorithm for learning a visual navigation policy that can quickly adapt to new observations induced by different camera configurations or different target objects. We assume the same action space across different robots; under this assumption, all agents are controlled using the same abstract navigation commands, such as go straight, turn left, turn right, and stop. Under this assumption, the key technical challenges here are to extract invariant knowledge shared across different observation spaces and to adapt the learned policy to a new scenario with a minimal amount of additional data.

We tackle this problem based on two steps: the baseline agent learning step and the adaptation step. During the baseline agent learning step, we train an effective navigation agent that consists of two components: 1) a perception network that encodes visual inputs into the latent variable and 2) an inference network that infers the cost-to-go value and optimal actions from the latent variable. During the adaptation phase, we only adapt the perception network using supervised learning (SL) while freezing the inference network. Because SL requires a lot of samples from the target environment that are not available for real-world applications, we propose a few-shot adaptation method via meta-learning.

We evaluate the proposed algorithm on a target-driven navigation problem on the Habitat simulator [5]. We investigate the adaptation of navigation skills to unseen scenarios with different camera heights and different target objects. Our 
experimental results indicate that the learned agent with one visual observation space can be quickly adapted to a new scenario by using less than five shots, which is enabled by our novel method of meta-learning on the latent space. We further validate the algorithm's design by comparing different hyper-parameters, the dimension of the latent parameters and the number of meta update steps.

\section{RELATED WORK}

\section{A. DRL based Visual Navigation}

Over the past few years, a tremendous effort has been put towards enabling autonomous navigation agents in realistic environments. For this purpose, many environments with various fidelity have been developed. The early work of Mirowski et al. [6] provides game-like maze environments, while recent simulators like MINOS [7], Habitat [5], and iGibson [8], [9], provide a physics-enabled simulated environment with a photo-realistic rendering for training and evaluation of autonomous agents performing various tasks such as object manipulation, navigation, and search. These simulators enable the agents to learn the task via trial-anderrors, over millions or billions of environment interactions, aiming for successful sim-to-real transfer.

Recently, Decentralized Distributed Proximal Policy Optimization (DD-PPO) [4] demonstrates that a near-perfect point-goal agent can be trained to navigate based on visual observations and GPS information in an end-to-end fashion. Yuke Zhu et al. [10] trains scene-specific expert policies while demonstrating how the control policy can be conditioned on visual target information, such as a picture of the goal location in addition to the agent's first-person view observation.

To enable longer-horizon navigation with spatio-temporal dependencies, the work of Fang [11] proposes a novel network architecture using Transformer [12] for visual navigation, which shows better performance than both reactive and Long short-term memory (LSTM) [13] agents. Active Neural SLAM [14], [15] shows that the careful hierarchical decomposition of the navigation policy leads to reduced sample complexity compared to typical end-to-end agents.

We utilize the Habitat simulator [5] and PPO [16] algorithm to train a vision-based target-driven expert policy. Our agent perceives the RGB visual information of the environment that contains a single visual target goal.

\section{B. Skill Generalization in Visual Navigation}

Various methods for generalizing the learned agents to new scenarios have been proposed [10], [17], [18], [19], [20], [21], [22]. One of the popular approaches to generalize over the various sensor and task configurations is Domain Randomization (DR) [19], [23], which trains an agent in randomized environments.

An alternative approach to address the generalization problem is a hierarchical approach that decomposes the problem into multiple sub-components. For instance, the object-search task can be decomposed into the detection of object-specific information and navigation based on the extracted information. The work of Ye et al. [17] jointly trains an object recognition model for predicting the object mask and a control policy. At the adaptation phase, only the object recognition model is adapted with supervised learning, while the control policy remains unchanged. Similarly, in the work of Devo et al. [18], the architecture is decomposed into two networks: object recognition network and navigation/exploration, where only the recognition part is adapted at the transfer stage. Yuke Zhu et al. [10] studies the generalization of RL in different scenes and tasks by sharing feature embeddings of perception network across scene-specific expert policies. With such design the perception network is ought to produce generalized features that are useful in different scenes.

Our policy is similarly decomposed into two components; however, we do not rely on any assumption regarding the task at hand. The expert policy (prior to adaptation) is trained in an end-to-end fashion with a constrain of a bottleneck between perception and inference components. We keep the inference network fixed at an adaptation stage and only adapt the perception part. While the task selected for experiments is related to navigation, our method can be applied to a wide range of vision-based control problems.

\section{Meta-learning and Few Shot Adaptation}

As opposed to learning a single policy exhibiting robust behaviors in all scenarios, researchers [24], [25], [26], [27], [28], [29] have developed algorithms to quickly adapt the learned policy to a new scenario, which is referred to as metalearning. One notable algorithm is Model-agnostic Metalearning (MAML) [24], which is a meta-learning algorithm that can be applied to both supervised learning and reinforcement learning. During the training phase of MAML, the optimizer brings the network parameters into the state where they are ready for fast adaptation to a new task. By taking a few gradient steps during the meta-adaptation phase, the agent becomes ready for the testing domain. In the work of Wortsman et al. [30], a self-supervised interaction objective is combined with MAML to enable the navigation agent to adapt throughout testing rollouts. Li et al. propose [31] to jointly meta-train a high-level policy with sub-skills in an unsupervised fashion and only adapt the high-level policy at the adaptation phase. Our approach also utilizes the MAML for training the perception network without relying on the learning of the sub-skills. We demonstrate that latent embedding enables rapid adaptation with only a handful of the target images set to the same configuration as in the source environment.

\section{A Few Shot Adaptation with Meta Learning}

In this section, we present our algorithm that adapts a learned visual navigation agent to a new observation function, which can be caused by different camera heights or different target objects. Our key idea is to train a baseline navigation agent with two components; the perception and inference networks connected by latent features, and to adapt 
only the perception network efficiently via meta-learning while freezing the inference network.

The rest of the section is organized as follows. We first describe the problem formulation (Section III-A) and explain the training of a baseline navigation agent (Section III-B). Then we describe fast adaptation with supervised learning (Section III-C) and meta learning (Section III-D).

\section{A. Problem Formulation}

Let us consider a target-driven visual navigation problem, where the agent aims to learn a policy to find the given target with egocentric visual inputs. We formulate this task as Partially Observable Markov Decision Processes (PoMDPs), $\left(\mathcal{S}, \mathcal{O}, \mathcal{A}, \mathcal{T}, r, p_{0}, \gamma\right)$, where $\mathcal{S}$ is the state space, $\mathcal{O}$ is the observation space, $\mathcal{A}$ is the action space, $\mathcal{T}$ is the transition function, $r$ is the reward function, $p_{0}$ is the initial state distribution and $\gamma$ is a discount factor.

In a typical navigation episode, at each time $t$, the agent receives an observation $\mathbf{o}_{t}$ based on current state $\mathbf{s}_{t}, \mathbf{o}_{t}=$ $c\left(\mathbf{s}_{t}\right)$. After the agent takes an action $\mathbf{a}_{t} \sim \pi\left(\mathbf{o}_{t}\right)$ based on the observation $\mathbf{o}_{t}$, it gets reward $r$ from the environment and arrives at a new state $\mathbf{s}_{t+1}=\mathcal{T}\left(\mathbf{s}_{t}, \mathbf{a}_{t}\right)$. The goal of reinforcement learning [32] is to find the optimal policy that maximizes the accumulated reward:

$$
J(\pi)=\mathbb{E}_{\mathbf{s}_{0}, \mathbf{a}_{0}, \ldots, \mathbf{s}_{T}} \sum_{t=0}^{T} \gamma^{t} r\left(\mathbf{s}_{t}, \mathbf{a}_{t}\right)
$$

Observation Space. In our problem, we assume that each robotic agent has its own observation function $\mathbf{o}_{t}=\tilde{c}\left(\mathbf{s}_{t}\right) \in$ $\tilde{\mathcal{O}}$, which represents its unique camera perspective, possibly with the target object class present in observation. Here, we regard $\mathbf{s}_{t}$ as the ground truth state at time $t$, which represents the agent's position and orientation. For instance, Turtlebot [33] and Fetch [34] robots have different camera heights of $0.15-0.55 \mathrm{~m}$ and $1.1-1.5 \mathrm{~m}$, which results in distinctive visual features in input images from the same location. Also, the observed visual inputs of different targets from the same position will be different from each other. We assume that an observation function $\tilde{c}$ typically returns a single RGB or RGB-D image. This robot-specific observation function defines our unique challenge of learning a common navigation policy that can be generalized to a new observation space.

Action Space. From the given observation $\mathbf{o}_{t}$, the agent takes an action $\mathbf{a}_{t} \in \mathcal{A}$, where the discrete action space $\mathcal{A}$ is defined as $\{$ MoveForward, TurnLeft, TurnRight, Stop $\}$, which is the default setting in the Habitat [5] environment. The episode is considered successful only if the agent calls the 'Stop' action at the location close enough to the predefined target. Otherwise, the episode is considered failed, and the agent does not receive a success reward.

Reward function. Our reward function is designed to encourage the agent to reach the goal, which is slightly adapted from the default setting in the Habitat:

$r_{t}\left(\mathbf{s}_{t}, \mathbf{a}_{t}\right)= \begin{cases}r_{s}, & \text { if } d_{t}^{g}<0.1 \text { and } \mathbf{a}_{t}=\text { Stop } \\ r_{d}+w_{1}\left(d_{t-1}^{g}-d_{t}^{g}\right)-w_{2}\left(d_{t-1}^{s}-d_{t}^{s}\right), \text { others }\end{cases}$

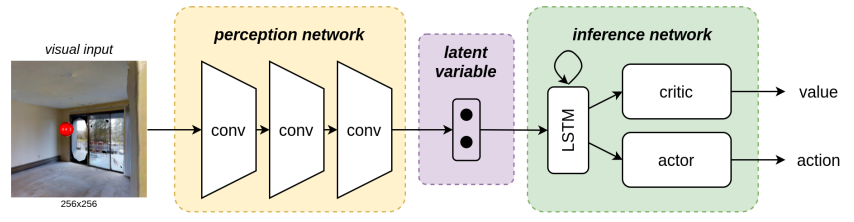

Fig. 2. A network architecture of a navigation agent, which consists of the perception and inference networks.

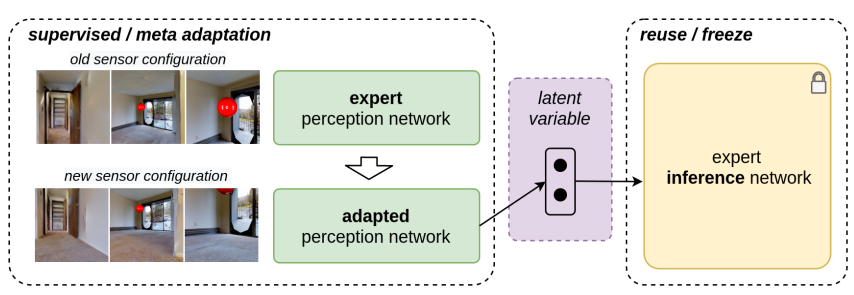

Fig. 3. Adaptation via supervised learning or meta learning. We only adapt the perception network while freezing the inference network.

where $r_{s}$ is the reward when the episode ends successfully, $r_{d}$ is a fixed negative live penalty, and $d_{t}^{g}$ and $d_{t}^{s}$ are the distances from the agent to the goal and start point at time $t$. The term $d_{t-1}^{s}-d_{t}^{s}$ is designed to encourage exploration at the beginning of episode to prevent the agent from being trapped in a narrow room. We set $r_{s}, r_{d}, w_{1}$, and $w_{2}$ as 10 , $-0.1,1$ and 0.1 for all the experiments.

\section{B. Navigation baseline}

As the first step, we train a baseline navigation agent that successfully finds an object based on visual inputs. Our algorithm particularly requires a highly effective agent because it defines the nominal behaviors of all the agents. We train a baseline agent with a standard configuration with the $1.25 \mathrm{~m}$ camera height with a red color target.

We use an end-to-end RL framework to train an agent to find a specified target with a single observation function. Our policy architecture consists of two components: the perception network and the inference network (Figure 2). For the perception network, we use a simple 3-layer CNN visual encoder to extract features, or the latent variables, of the RGB image input. The inference network processes the features through a Long Short-Term Memory network (LSTM) [13] and computes the final actions and values. We optimize the entire policy network using Proximal Policy Optimization (PPO) algorithm [16].

\section{Adaptation with Supervised Learning}

Once we obtain a baseline agent, the next step is to adapt it to an updated observation function $\mathbf{o}_{t} \sim \tilde{c}\left(\mathbf{s}_{t}\right)$ that is different from the original function $c$. A naive solution is to train a new policy from scratch; however, this approach takes a lot of computational time and does not guarantee that the same hyperparameters can learn a satisfactory policy in a new scenario. Alternatively, a more structured approach is domain randomization (DR) [19], [23], where we train a single navigation agent while randomizing observation 
functions during the training process. However, DR can learn a conservative policy that is suboptimal for many tasks.

Instead, we propose only to adapt the perception network to a new observation function $\tilde{c}$ using supervised learning (Figure 3), which is similar to the idea of the prior work of Gordon et al. [22]. More specifically, we adapt the learned perception network to produce the same latent variables from the collected images, which will be the inputs to the inference network. We do not change the inference network because the agent can use the same actions to solve the problem if the current state is inferred correctly.

For all the states $\left\{\mathbf{s}_{1}, \cdots, \mathbf{s}_{K}\right\}$ in the sampled trajectories, we collect all the tuples of $\left(c\left(\mathbf{s}_{t}\right), \tilde{c}\left(\mathbf{s}_{t}\right), \phi\left(\mathbf{o}_{t}\right)\right)$ where $\phi$ is the perception network that outputs the latent state from the visual observations. The same simulator state $s_{t}$ is used as input to both $c$ and $\tilde{c}$, and the difference in $o_{t}$ is reflected in the sensory observation (e.g. camera view from a different sensor heights or a distinct target object). Then we can train a new perception function $\tilde{\phi}\left(\mathbf{s}_{t}\right)$ by minimizing the loss $\mathcal{L}=\sum\left|\phi\left(c\left(\mathbf{s}_{t}\right)\right)-\tilde{\phi}\left(\tilde{c}\left(\mathbf{s}_{t}\right)\right)\right|$. Our preliminary experiment indicates that this adaptation with SL works well for a target navigation task in various scenarios given enough data from the new environment.

\section{Adaptation with Meta-Learning}

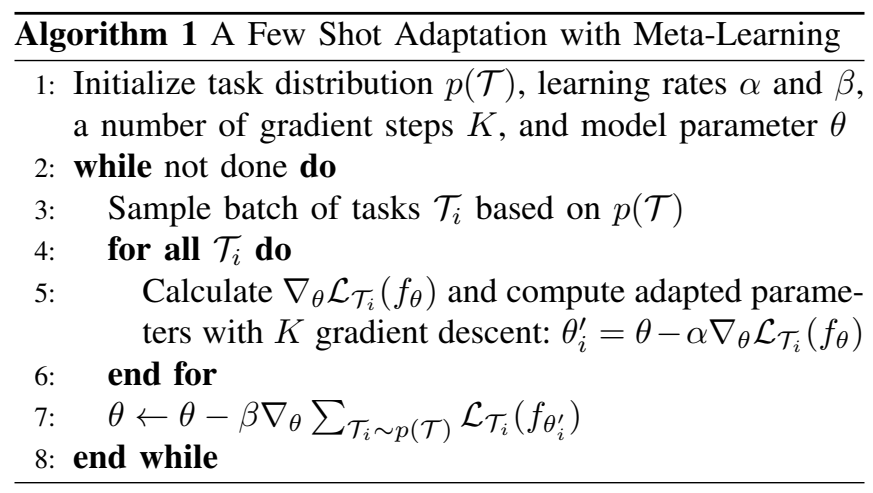

However, if we aim to apply the SL approach to real-world scenarios, it is likely to be infeasible because it requires a lot of samples from the target-environment. In addition, it requires us to collect the observation $\tilde{c}\left(\mathbf{s}_{t}\right)$ from the same simulation trajectory $\left\{\mathbf{s}_{1}, \cdots, \mathbf{s}_{K}\right\}$. But it is difficult for a robot to exactly track the given simulation trajectory in the real-world environment due to the difference in observation functions and control errors, unless we exploit other frameworks, such as Simultaneous Localization and Mapping (SLAM) [35] or external motion capture systems.

Instead, it will be more data-efficient if we can adapt to a new scenario using only a handful of examples via metalearning. In our scenario, this corresponds to collection of observations from a few key places, such as the four corners of the room or locations near the goal object. Please note that we simplified the problem by assuming that the sim-toreal gap is only introduced by a new observation function, while the other sim-to-real factors have minimal impacts due to the photo-realistic rendering of the environment.
We propose a data-efficient adaptation via meta-learning, which requires only a few shots. We exploit the existing meta-learning framework, Model Agnostic Meta Learning [24], which explicitly learns to make a quick adaptation to the perception network $\tilde{\phi}$ with a small number of gradient steps. In our scenario, we consider a distribution of tasks, $p(\mathcal{T})$, where each task $\mathcal{T}_{i}$ is to navigate with the given observation function $\tilde{c}_{i}$. We train our meta-perception network $\tilde{\phi}_{\theta}$, which is parameterized by $\theta$, to learn an unseen task from only $M \leq 10$ samples. To this end, we first sample a batch of $N$ tasks $\mathcal{T}_{1} \ldots N$ from the distribution and compute the adapted parameters $\theta_{1} \cdots N$ by taking $K$ gradient steps. Then we update the meta-parameters $\theta$ by aggregating the changes of the adapted parameters, $\theta_{1 \cdots N}$. The algorithm is summarized in Algorithm 1. For more details, please refer to the original paper [24].

\section{EXPERIMENTAL EVALUATION}

In this section, we discuss the simulation experiments for validating the proposed algorithm. We design the experiments to answer the following questions.

1) Can our algorithm effectively adapt to unseen observation functions?

2) Does our meta-adaptation show better generalization than other algorithms?

3) How does the selection of hyper-parameters impact the performance?

\section{A. Experiment Setup}

We use the Habitat simulator as our testing platform, which offers photo-realistic rendering at high speed, up to over several thousand FPS on a single GPU. All the experiments are conducted on a laptop equipped with an $8 \mathrm{~GB}$ Intel i7 8750H Processor and a GTX1060 GPU. We firstly train the navigation baseline (Section III-B) in the Habitat Avonia environment to find a red ball with the $1.25 \mathrm{~m}$ camera height. The training dataset contains 4000 episodes, and the validation set contains 200 new episodes with randomized initial agent positions and target object locations.

We learn policies using PPO [16], where the discount factor is set to 0.99 , and the GAE parameter is 0.95 . After 6 hours of training, the agent could navigate to the target with $84 \%$ success rate and 0.69 success weighted path length (SPL) [36]. For adaptation wiht meta learning, our MAML implementation uses the same inner learning rate of $2 \mathrm{e}-4$ and outer learning rate of $2 \mathrm{e}-4$, three-shots(all containing the object), and ten gradient step updates.

\section{B. Adaptation Experiments with Meta-Learning}

Figure 4 shows the rollouts of three agents:

1) Navigation Baseline: The agent is trained and evaluated with fixed sensor $(1.25 \mathrm{~m})$ and target configuration.

2) Sensor Height Changing: The agent is meta-trained with the heights between $0.6 \mathrm{~m}$ and $1.3 \mathrm{~m}$ and evaluated at $1.8 \mathrm{~m}$ after meta-adaptation. 


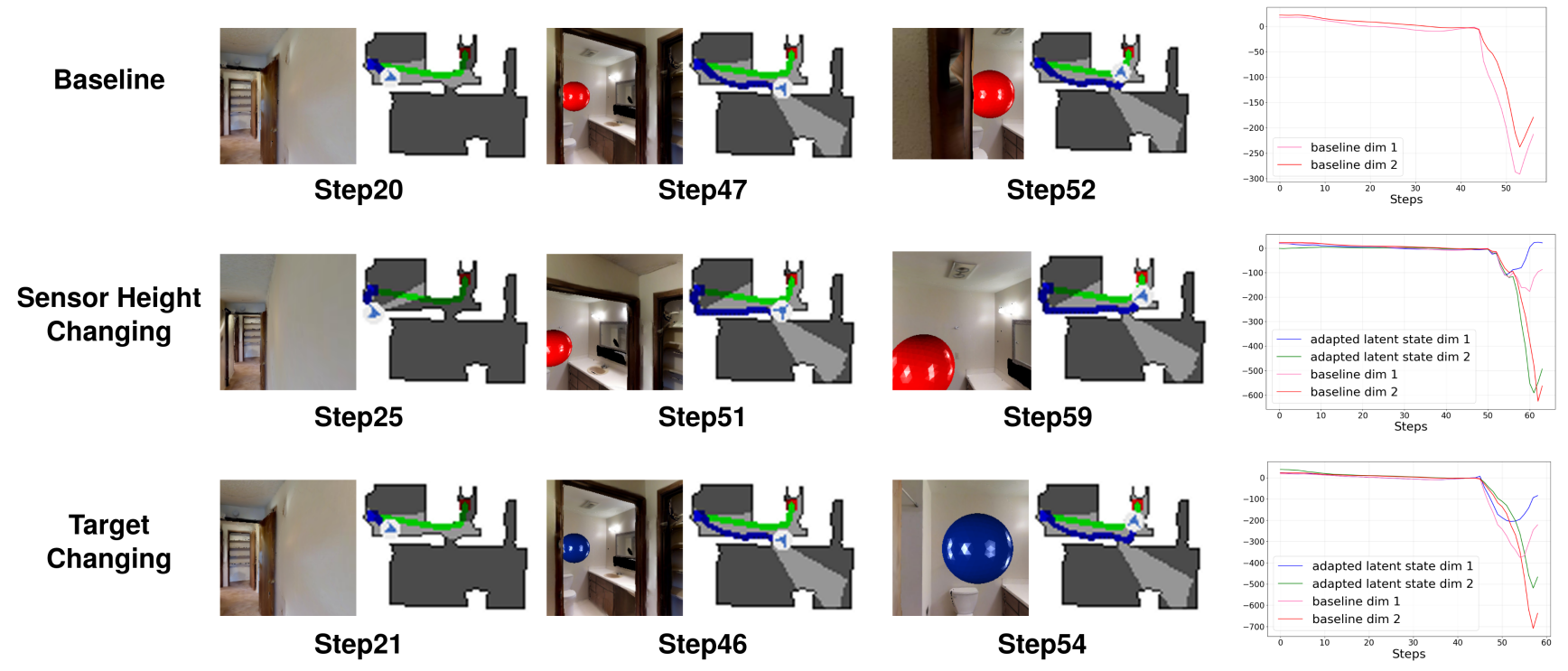

Fig. 4. Learned Behaviors of the baseline agent with the default setting (Top) and the adapted agent with meta-learning for the camera height change (Middle) and the target object change (Bottom). We show three observations and three map images, which are sampled at different moments. For all experiments, green lines indicate the shortest path between the starting and goal locations. The rightmost column represents the trajectories of two dimensional latent variables over time.
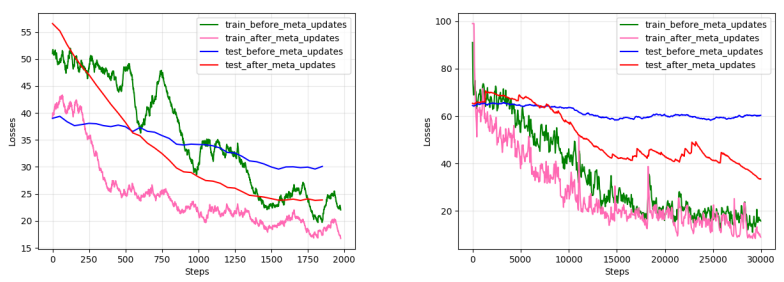

Fig. 5. Learning curves of meta-adaptation for two tasks: sensor height changes (Left) and object color changes (Right). The results indicate that meta learning can successfully adapt to new scenarios (blue to red).

3) Target Changing: The agent is meta-trained with 10 target colors and evaluated with a new dark-blue color after meta-adaptation.

Each row contains three egocentric observations and three map images collected at three different moments: when an agent is searching the ball, locating the ball, and getting close to the ball, respectively. As shown in Figure 4, our algorithm successfully adapts the policy to new scenarios using only three-shots. As expected, the adapted agents show very similar behaviors to the baseline agent because we only adapt the perception network. The rightmost column of Figure 4 plots two-dimensional latent values in the rollouts, which become larger when the agent detects the target object. Metaadapted agents in both scenarios closely track the baseline agent's latent features, and are able to call the "STOP" action to end the episode successfully.

Figure 5 illustrates the learning curves of two metaadapted agents. The training takes only 15 minutes for the sensor height changing scenario while taking three hours for the color-changing problem. For both scenarios, the testing loss after meta-updates is much lower than the testing loss before beta-updates, highlighting the importance of the metaupdates in our algorithm. And we also want to note that the testing loss for the color-changing problem is still going down even when the training curve is saturated.

We compare the proposed algorithm against the navigation baseline and a few other training methods:

1) Navigation Baseline: The agent is trained with a nominal observation function as described in Section $\amalg I-B$.

2) Domain Randomization: The agent is trained with randomized observation functions within the training range.

3) Domain Randomization with target embedding: Inspired by the work of Zhu et al. [10], we amend domain randomization to take an observation of the target as additional inputs. Such augmentation serves as an oversampling mechanism that balances the distribution of target and non-target images.

4) Supervised Learning (SL): The agent is adapted with supervised learning as described in Section III-C

5) SL + Few Shots: Based on the perception network learned by SL, we further refine the model by leveraging three images from the target environment, which uses the same amount of data as meta-learning.

6) MAML + Few Shot Adaptation The agent is trained using the method described in Section III-D and task settings described in Section IV-B

We apply these methods to two tasks: camera sensor height 
TABLE I

COMPARISON OF ADAPTATION Algorithms

\begin{tabular}{lllll}
\hline & \multicolumn{2}{c}{ Training } & \multicolumn{2}{c}{ Testing } \\
\cline { 2 - 6 } Task: Camera Height Changes & SPL & Success & SPL & Success \\
\hline Navigation Baseline & 0.69 & 0.84 & 0.00 & 0.00 \\
Domain Randomization & $\mathbf{0 . 7 1}$ & $\mathbf{0 . 8 6}$ & 0.04 & 0.10 \\
DR + target embedding & 0.69 & 0.82 & 0.06 & 0.09 \\
SL & 0.66 & 0.83 & 0.02 & 0.05 \\
SL + Few Shots & 0.61 & 0.75 & 0.01 & 0.03 \\
MAML + Few Shot Adaptation & 0.67 & 0.84 & $\mathbf{0 . 1 8}$ & $\mathbf{0 . 2 5}$ \\
\hline \hline & \multicolumn{2}{c}{ Training } & & Testing \\
\cline { 2 - 6 } & SPL & Success & SPL & Success \\
Task: Target Color Changes & $\mathbf{0 . 6 9}$ & $\mathbf{0 . 8 4}$ & 0.06 & 0.10 \\
\hline Navigation Baseline & 0.41 & 0.50 & 0.16 & 0.21 \\
Domain Randomization & 0.43 & 0.52 & 0.16 & 0.22 \\
DR + target embedding & 0.63 & 0.82 & 0.18 & 0.24 \\
SL & 0.60 & 0.78 & 0.08 & 0.11 \\
SL + Few Shots & 0.63 & 0.80 & $\mathbf{0 . 5 0}$ & $\mathbf{0 . 7 0}$ \\
MAML + Few Shot Adaptation & & & & \\
\hline
\end{tabular}
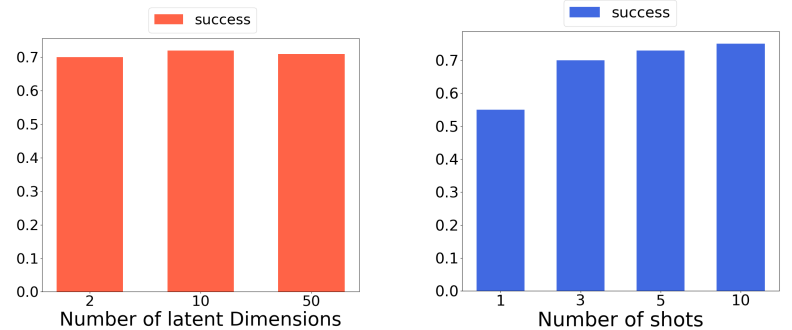

Fig. 6. Analysis on meta-learning adaptation. We report the average success rates that are measured in both sensor height changing and target color changing scenarios. We investigate the number of the latent dimension (Left) and the number of shots (Right). More shots lead to better success rates but the number of latent dimensions do not have significant impacts on the success rate.

changes task and target color changes task. In both tasks, the training and testing range of either method are the same. For all experiments, we train each policy until they are fully converged. We did not set the same computation budgets because some methods (Navigation Baseline, Domain Randomization) are pure reinforcement learning and the others combine reinforcement learning and supervised learning. We run 200 episodes for each agent and take the average SPL and success rate to ensure the accuracy.

\section{Comparison of Adaptation Algorithms}

The results are summarized in Table I During the training, all of the methods produce approximately equal results, and all perform on par with the Navigation Baseline. However, in the testing setting our algorithm, adaption with meta-learning shows the best performance in both scenarios, while others approaches perform poorly on the unseen tasks. In the color changing case, our algorithm dramatically outperforms other methods, and the SPL and success rate are close to that of baseline agent in the training agent.

\section{Analysis}

We further analyze the proposed algorithm by conducting an ablation study. Particularly, we investigate the impact of two hyperparameters: the dimension of the latent space between the perception and inference networks, which is studied based on three-shot MAML adaptation, and the number of shots in meta-adaptation studied with two-dimensional latent space. Intuitively, the dimension of the latent space is expected to have an impact on the performance: a smaller dimension may be easier to adapt but also may result in a more convoluted latent space. We also expect that more shots grant the meta-learning process more adaptation capability while making the entire network harder to learn.

We compare the performance of meta-learning with different hyperparameters in Figure 6 All of the experiments are evaluated in the testing sets for both sensor height changing and object color-changing tasks. Against our intuition, the dimension of the latent space does not significantly affect the success rate. We suspect that this is because a lowdimensional embedding is sufficient for our testing scenarios: please note that an agent can achieve the $70 \%$ success rate with only two-dimensional latent features. Then a high dimensional space is expected to have a similar embedding structure, which is also easy to learn. On the other hand, the number of shots positively impacts the success rate: one-shot adaptation results in only a 55\% success rate while the tenshot adaptation achieves a $73 \%$ success rate. However, more shots require us to collect more real-world data, which can be cumbersome on real robots. We choose three shots for all the experiments, which shows near the optimal performance with the minimal number of additional examples.

\section{CONCLUSION}

We showed that meta-learning on an embedding space produced by a visual encoder demonstrates promising results at the adaptation of the navigation agents in the new sensory configurations. We effectively apply our method to two different adaptation scenarios, object color and sensor height changing problems, in the target-driven visual navigation task. Our method requires only a handful of shots from the target environments and outperforms the baseline generalization methods described in the paper.

When we consider the fact that only a few shots are required at the adaptation stage, our method could potentially be effective in the sim-to-real transfer. In this approach, the agent will be firstly trained in the simulated environment utilizing the scalability of the recent deep RL algorithms, without the safety risk to the robot or its surroundings. Then the learned policy can be adapted to the real-world scenario via the proposed meta-adaptation algorithm, which requires only a few real-world images. We plan to further investigate our algorithm on real robots, such as Fetch [34] or TurtleBot [33].

While we evaluate our algorithm on two different scenarios, there exists a variety of sensory adaptation scenarios that can be further tested in the future. Such variations include environment lighting, camera properties (FoV, lens distortions), multiple cameras, different sensors (LIDAR and IMUs), more complex object shapes, and the combination of all of these factors. 


\section{REFERENCES}

[1] S. Jung, S. Hwang, H. Shin, and D. H. Shim, "Perception, guidance, and navigation for indoor autonomous drone racing using deep learning," IEEE Robotics and Automation Letters, vol. 3, no. 3, pp. 25392544, 2018.

[2] H.-T. L. Chiang, J. Hsu, M. Fiser, L. Tapia, and A. Faust, "Rl-rrt: Kinodynamic motion planning via learning reachability estimators from rl policies," IEEE Robotics and Automation Letters, vol. 4, no. 4, pp. 4298-4305, 2019.

[3] A. Amini, G. Rosman, S. Karaman, and D. Rus, "Variational end-toend navigation and localization," in 2019 International Conference on Robotics and Automation (ICRA). IEEE, 2019, pp. 8958-8964.

[4] E. Wijmans, A. Kadian, A. Morcos, S. Lee, I. Essa, D. Parikh, M. Savva, and D. Batra, "Dd-ppo: Learning near-perfect pointgoal navigators from 2.5 billion frames," arXiv, pp. arXiv-1911, 2019.

[5] Manolis Savva*, Abhishek Kadian*, Oleksandr Maksymets*, Y. Zhao, E. Wijmans, B. Jain, J. Straub, J. Liu, V. Koltun, J. Malik, D. Parikh, and D. Batra, "Habitat: A Platform for Embodied AI Research," in Proceedings of the IEEE/CVF International Conference on Computer Vision (ICCV), 2019.

[6] P. Mirowski, R. Pascanu, F. Viola, H. Soyer, A. J. Ballard, A. Banino, M. Denil, R. Goroshin, L. Sifre, K. Kavukcuoglu et al., "Learning to navigate in complex environments," arXiv preprint arXiv:1611.03673, 2016.

[7] M. Savva, A. X. Chang, A. Dosovitskiy, T. Funkhouser, and V. Koltun, "MINOS: Multimodal indoor simulator for navigation in complex environments," arXiv:1712.03931, 2017.

[8] F. Xia, A. R. Zamir, Z.-Y. He, A. Sax, J. Malik, and S. Savarese, "Gibson env: real-world perception for embodied agents," in Computer Vision and Pattern Recognition (CVPR), 2018 IEEE Conference on. IEEE, 2018

[9] F. Xia, W. B. Shen, C. Li, P. Kasimbeg, M. E. Tchapmi, A. Toshev, R. Martín-Martín, and S. Savarese, "Interactive gibson benchmark: A benchmark for interactive navigation in cluttered environments," IEEE Robotics and Automation Letters, vol. 5, no. 2, pp. 713-720, 2020.

[10] Y. Zhu, R. Mottaghi, E. Kolve, J. J. Lim, A. Gupta, L. Fei-Fei, and A. Farhadi, "Target-driven visual navigation in indoor scenes using deep reinforcement learning," in 2017 IEEE international conference on robotics and automation (ICRA). IEEE, 2017, pp. 3357-3364.

[11] K. Fang, A. Toshev, L. Fei-Fei, and S. Savarese, "Scene memory transformer for embodied agents in long-horizon tasks," in Proceedings of the IEEE Conference on Computer Vision and Pattern Recognition, 2019, pp. 538-547.

[12] A. Vaswani, N. Shazeer, N. Parmar, J. Uszkoreit, L. Jones, A. N. Gomez, Ł. Kaiser, and I. Polosukhin, "Attention is all you need," Advances in neural information processing systems, vol. 30, pp. 59986008, 2017.

[13] S. Hochreiter and J. Schmidhuber, "Long short-term memory," Neural computation, vol. 9, no. 8, pp. 1735-1780, 1997.

[14] D. S. Chaplot, D. Gandhi, S. Gupta, A. Gupta, and R. Salakhutdinov, "Learning to explore using active neural slam," arXiv preprint arXiv:2004.05155, 2020.

[15] D. S. Chaplot, D. P. Gandhi, A. Gupta, and R. R. Salakhutdinov, "Object goal navigation using goal-oriented semantic exploration," Advances in Neural Information Processing Systems, vol. 33, 2020.

[16] J. Schulman, F. Wolski, P. Dhariwal, A. Radford, and O. Klimov, "Proximal policy optimization algorithms," arXiv preprint arXiv:1707.06347, 2017.

[17] X. Ye, Z. Lin, H. Li, S. Zheng, and Y. Yang, "Active object perceiver: Recognition-guided policy learning for object searching on mobile

[20] A. Sax, B. Emi, A. R. Zamir, L. Guibas, S. Savarese, and J. Malik, "Mid-level visual representations improve generalization and sample efficiency for learning visuomotor policies," arXiv preprint arXiv:1812.11971, 2018. robots," in 2018 IEEE/RSJ International Conference on Intelligent Robots and Systems (IROS). IEEE, 2018, pp. 6857-6863.

[18] A. Devo, G. Mezzetti, G. Costante, M. L. Fravolini, and P. Valigi, "Towards generalization in target-driven visual navigation by using deep reinforcement learning," IEEE Transactions on Robotics, vol. 36 , no. 5, pp. 1546-1561, 2020

[19] J. Tobin, R. Fong, A. Ray, J. Schneider, W. Zaremba, and P. Abbeel, "Domain randomization for transferring deep neural networks from simulation to the real world," in International Conference on Intelligent Robots and Systems (IROS). IEEE, 2017, pp. 23-30.

[21] A. Mousavian, A. Toshev, M. Fišer, J. Košecká, A. Wahid, and J. Davidson, "Visual representations for semantic target driven navigation," in 2019 International Conference on Robotics and Automation (ICRA). IEEE, 2019, pp. 8846-8852.

[22] D. Gordon, A. Kadian, D. Parikh, J. Hoffman, and D. Batra, "Splitnet: Sim2sim and task2task transfer for embodied visual navigation," in Proceedings of the IEEE International Conference on Computer Vision, 2019, pp. 1022-1031.

[23] F. Sadeghi and S. Levine, "Cad2rl: Real single-image flight without a single real image," arXiv preprint arXiv:1611.04201, 2016.

[24] C. Finn, P. Abbeel, and S. Levine, "Model-agnostic meta-learning for fast adaptation of deep networks," arXiv preprint arXiv:1703.03400, 2017.

[25] A. Nichol, J. Achiam, and J. Schulman, "On first-order meta-learning algorithms," arXiv preprint arXiv:1803.02999, 2018.

[26] Y. Yang, K. Caluwaerts, A. Iscen, J. Tan, and C. Finn, "Norml: Noreward meta learning," in Proceedings of the 18th International Conference on Autonomous Agents and MultiAgent Systems. International Foundation for Autonomous Agents and Multiagent Systems, 2019, pp. 323-331.

[27] K. Rakelly, A. Zhou, C. Finn, S. Levine, and D. Quillen, "Efficient offpolicy meta-reinforcement learning via probabilistic context variables,' in International conference on machine learning. PMLR, 2019, pp. 5331-5340

[28] A. A. Rusu, D. Rao, J. Sygnowski, O. Vinyals, R. Pascanu, S. Osindero, and R. Hadsell, "Meta-learning with latent embedding optimization," arXiv preprint arXiv:1807.05960, 2018.

[29] M. Yin, G. Tucker, M. Zhou, S. Levine, and C. Finn, "Meta-learning without memorization," arXiv preprint arXiv:1912.03820, 2019.

[30] M. Wortsman, K. Ehsani, M. Rastegari, A. Farhadi, and R. Mottaghi, "Learning to learn how to learn: Self-adaptive visual navigation using meta-learning," in Proceedings of the IEEE Conference on Computer Vision and Pattern Recognition, 2019, pp. 6750-6759.

[31] J. Li, X. Wang, S. Tang, H. Shi, F. Wu, Y. Zhuang, and W. Y. Wang, "Unsupervised reinforcement learning of transferable meta-skills for embodied navigation," in Proceedings of the IEEE/CVF Conference on Computer Vision and Pattern Recognition, 2020, pp. 12 123-12 132.

[32] R. S. Sutton and A. G. Barto, Reinforcement learning: An introduction. MIT press Cambridge, 1998, vol. 1.

[33] W. Garage, "What is a turtlebot?" [Online]. Available: http: //turtlebot.com/

[34] M. Wise, M. Ferguson, D. King, E. Diehr, and D. Dymesich, "Fetch and freight: Standard platforms for service robot applications," in Workshop on autonomous mobile service robots, 2016.

[35] T. Bailey and H. Durrant-Whyte, "Simultaneous localization and mapping (slam): Part ii," IEEE robotics \& automation magazine, vol. 13, no. 3, pp. 108-117, 2006.

[36] P. Anderson, A. Chang, D. S. Chaplot, A. Dosovitskiy, S. Gupta, V. Koltun, J. Kosecka, J. Malik, R. Mottaghi, M. Savva et al., "On evaluation of embodied navigation agents," arXiv preprint arXiv:1807.06757, 2018 